\title{
Erratum
}

\section{Erratum: 100-Year-Old Vaccine for a Novel Virus: Killing Two Birds with One Stone!}

In the article titled "100-Year-Old Vaccine for a Novel Virus: Killing Two Birds with One Stone!", published on pages 291-294, Issue 3, Volume 41 of Indian Journal of Medical and Paediatric Oncology ${ }^{[1]}$, the affiliations of authors and corresponding author address detail are incorrectly written as

Chetan Dhamne ${ }^{1}$, Gagan Prakash², Gaurav Narula ${ }^{1}$, Shripad Banavali ${ }^{1}$, Jyoti Bajpai ${ }^{1}$

${ }^{1}$ Department of Medical Oncology, TMC, Mumbai, Maharashtra, India

${ }^{2}$ Department of Surgical Oncology, TMC, Mumbai, Maharashtra, India

Address for correspondence: Dr. Chetan Dhamne, Department of Medical Oncology, TMC, Mumbai, Maharashtra, India. E-mail: chetandhamne@gmail.com

The correct affiliations of authors and corresponding address details should read as

Chetan Dhamne', Gagan Prakash², Gaurav Narula', Shripad Banavali¹, Jyoti Bajpai ${ }^{1}$

${ }^{1}$ Department of Medical Oncology, Tata Memorial Centre, Homi Bhabha National Institute, Mumbai, Maharashtra, India ${ }^{2}$ Department of Surgical Oncology, Tata Memorial Centre, Homi Bhabha National Institute, Mumbai, Maharashtra, India

Address for correspondence: Dr. Chetan Dhamne, Department of Medical Oncology, Tata Memorial Centre, Homi Bhabha National Institute, Mumbai, Maharashtra, India. E-mail: chetandhamne@gmail.com

\section{Reference}

1. Dhamne C, Prakash G, Narula G, Banavali S, Bajpai J. 100-year-old vaccine for a novel virus: Killing two birds with one stone! Indian J Med Paediatr Oncol 2020;41:291-4.

DOI: 10.4103/0971-5851.293847 